\title{
Reactions of Mongolian gerbils in the presence of urine stimuli
}

\author{
TERRY F. PETTIJOHN \\ Ohio State University, Marion, Ohio 43302
}

\begin{abstract}
Forty sexually mature Mongolian gerbils served as subjects in a study designed to examine responsiveness to samples of urine. In a two-choice comparison situation in the home cage, each isolated gerbil was tested for amount of time spent with each urine sample during a 3-min period. Male gerbils spent significantly more time with estrus female urine than nonestrus female gerbil urine, and with nonestrus female gerbil urine than with either male gerbil urine or water. Female gerbils did not respond differentially to any of the stimuli. Neither sex discriminated between gerbil and hamster urine of either sex. Results suggest that female gerbil urine communicates sexual identity and, especially, sexual readiness to the male gerbil.
\end{abstract}

Urine is an important source of intraspecific information in several rodents, the most extensively studied of which have been rats and guinea pigs. Calhoun (1962) observed that wild male rats appeared to detect the sexual state of females in natural situations. Lydell and Doty (1972) tested Calhoun's ideas in the laboratory and found that sexually experienced male rats preferred estrus urine odor to nonestrus urine odor or distilled water in a two-choice situation. Beauchamp (1973) tested adult male guinea pigs' preferences to urine of various sources, and found that adult female guinea pig urine was preferred to urine of adult males. These studies indicate that urine can convey information on sexual identification to conspecifics.

The present research was designed to investigate the reactions of gerbils in the presence of urine stimuli. Although Dagg and Windsor (1971) found that several young gerbils could be trained in a $T$ maze to discriminate between various urine odors, they did not investigate preference or other reactions. Halpin (1974) found that male gerbils were capable of discriminating between individual male gerbil urine odors, suggesting a function of individual identification.

Most attention in gerbil research has been focused on olfactory communication conveyed through the marking of objects with the midventral sebaceous gland (Thiessen, Friend, \& Lindzey, 1968). Nyby, Thiessen, and Wallace (1970) found that gerbils avoided odors from other gerbils in a Y-tube preference test, while Baran (1973) found that gerbils were attracted to odors or objects marked by other gerbils.

Reprint requests should be sent to Terry F. Pettijohn, Department of Psychology, Ohio State University, Marion, Ohio 43302. The author wishes to thank J. Panksepp, C. Mynatt, S. Vessey, and especially J, P. Scott for their helpful comments on the manuscript.
In many of the previous gerbil studies, observed reaction was to confounded sources of odor (urine, feces, sebum), and thus it is unclear what information may be communicated specifically via urine. As a step toward determining the function of urine in gerbils' social system, the present research investigated the responsiveness of isolated gerbils to two samples of urine presented simultaneously in the home cage.

\section{METHODS}

\begin{abstract}
Subjects
Twenty male and 20 female adult Mongolian gerbils (Meriones unguiculatus) between the ages of 6 and 18 months served as subjects. All subjects were housed with littermates until approximately 3 months of age, when they were separated into nonrelated groups of four (two of each sex). A week prior to the beginning of the study, each subject was isolated in a large opaque plastic laboratory cage $(24 \times 45 \times 12 \mathrm{~cm})$ with a wire top. Bedding, $1 \mathrm{~cm}$ deep, covered the cage floor, and food and water were always available.
\end{abstract}

\section{Test Stimuli}

For each of the 40 subjects, the following test comparisons were made. Each subject was tested once on each of the six comparisons, receiving one comparison test per day, with at least 1 day intervening between comparisons. To determine whether gerbils respond differentially to urine from their own species and to urine from another, "species comparisons" were made of: (a) female gerbil urine vs. female hamster (Mesocricetus auratus) urine, and (b) male gerbil urine vs. male hamster urine. To determine whether gerbils respond differentially to urine from oppositesexed and like-sexed conspecifics, a "sex comparison" was made of female gerbil urine vs. male gerbil urine. To determine whether gerbils respond differentially to female gerbils that are in estrus, a "sexual-state comparison" was made between urine from estrus gerbils vs. urine from nonestrus gerbils. To determine the strength of responsiveness to gerbil urine, "control comparisons" were made of: (a) female gerbil urine vs. water, and (b) male gerbil urine vs. water. Urine was obtained from nonsubject donors by picking the animal up and, while holding it over a clean plexiglas sheet, placing a small can of ether under its nose. The urine was then recovered with a clean eyedropper. Urine 
from two donors was usually pooled and was always used in testing within $1 \mathrm{~h}$ of collection.

All female test urine was obtained from nonestrus females except for the gerbil sexual-state comparison. Estrus was determined by systematically pairing the female donor gerbils and hamsters with reliable indicator males after the urine was obtained (after Barfield, \& Beeman, 1968). A female subject's data were included only when she was in the nonestrus state, which was determined after the day's testing. In the sexual-state comparison, estrus was induced in nonestrus gerbils by injecting 6 mcg estradiol benzoate followed $36 \mathrm{~h}$ later by $0.5 \mathrm{mg}$ progesterone. Receptivity occurred in about $9 \mathrm{~h}$ (following techniques of Kuehn \& Zucker, 1968).

\section{Testing Procedure}

For each test stimulus, approximately $0.2 \mathrm{ml}$ of liquid was placed on a new sterile microscope glass slide. Two fresh samples, each on a different slide, were presented for each comparison. The home cage was divided into six equal sections, $12 \times 15 \mathrm{~cm}$ (marked by vertical lines on the walls), and the samples were placed (counterbalanced) in the centers of the two sections at one end. The subject was always in one of the four remaining sections at the beginning of each test. Data were recorded for $3 \mathrm{~min}$, after which the slides were removed. The main dependent variable was the number of seconds (180 maximum) spent in each section in which a stimulus was placed. Also recorded were: location (section) of the subject every $15 \mathrm{sec}$, and frequencies of defecation, urination, and ventral marking.

\section{RESULTS}

In a typical 3-min observation, the subject ran to first one stimulus and then the other, sniffing, pawing and licking each urine drop in turn, and ran about the cage, investigating the bedding thoroughly. It ran to each stimulus several times, usually spending more time with a particular one at each approach. Often the subject would touch the urine and then groom itself, usually while remaining in the same section of the cage.

For each comparison in each sex group, a t test for nonindependent subjects was run on the time (number of seconds) each subject spent in each section containing a stimulus. Figure 1 shows for all six comparisons the mean times spent by male gerbils in the stimulus-containing sections. The greatest difference in responsiveness occurred in the comparison between estrus female gerbil urine and that of nonestrus female gerbils $[\mathrm{t}(19)=3.10, \mathrm{p}<.01]$, with the subject spending more time with urine from the estrus female gerbils. The female gerbil urine vs. male gerbil urine comparisons $[\mathrm{t}(19)=2.43, \mathrm{p}<$ $.025]$ and the female gerbil urine vs. water comparison $[\mathrm{t}(19)=2.95, \mathrm{p}<.01]$ also showed statistically significant differences, with the subject spending more time with female gerbil urine in each case. The remaining three comparisons did not reach significance for the male subjects.

Figure 2 shows the mean times spent by female gerbil subjects in the stimulus-containing sections. In contrast with the male subject data, none of the comparisons showed statistically significant differences. The female gerbils spent roughly 20 to $30 \mathrm{sec}$

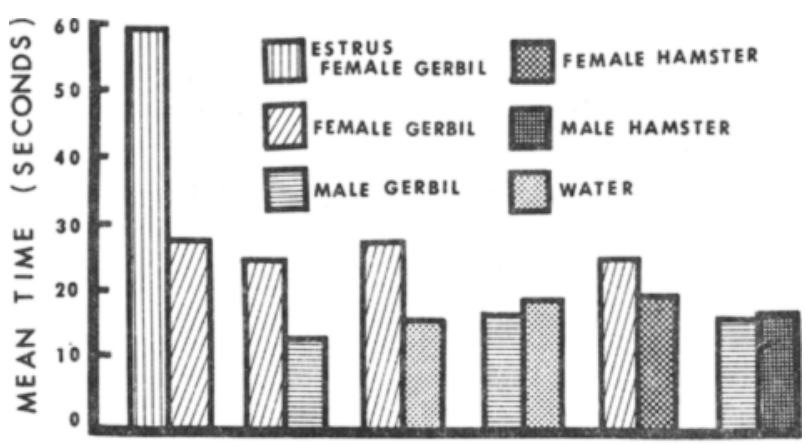

STIMULUS SOURCE

Figure 1. Mean times spent by male subjects near each test stimulus in each comparison.

with each stimulus in each comparison and did not spend much more time with any particular sample.

A statistical analysis was not run on the frequencies of ventral marking, urination, or defecation since they were so low (means less than two per subject in any comparison with many means less than one per subject). Overall, the males marked more than the females, but the defecation and urination scores were similarly low.

To determine whether the subjects had randomly moved about the cage or responded strongly toward or away from the two urine test stimuli, a Friedman two-way analysis of variance with ranks was run on the frequencies of $15-\mathrm{sec}$ location scores for each section. Sections 1 and 2 were combined, as were 3 and 4 , and 5 and 6 , so that the frequencies from the combined sections (thirds of the cage) could be ranked for each subject in each of the comparisons. The only statistically significant comparisons were for male subjects presented urine from estrus female gerbils vs. nonestrus female gerbils $\left[\chi_{r}^{2}(2)=10.2\right.$, $\mathrm{p}<.01]$ and female gerbils vs. water $\left[\chi_{\mathrm{r}}^{2}(2)=10.4\right.$, $\mathrm{p}<.01]$. In these two comparisons, the male gerbils had higher location scores for the third of the cage nearest the stimuli.

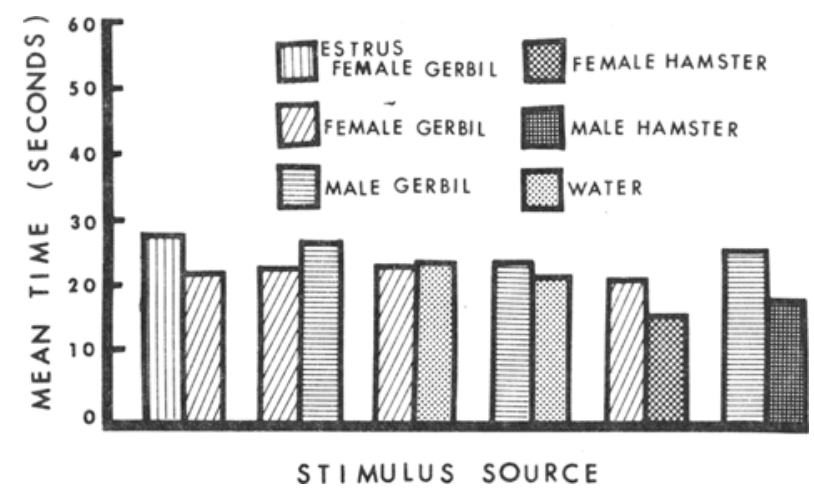

Figure 2. Mean times spent by female subject near each test stimulus in each comparison. 


\section{DISCUSSION}

This research demonstrates that the reactions of gerbils to urine samples can be studied by the use of a two-choice situation. The subjects investigated each urine source and were able to move freely about their home cage. The time spent with each urine source, shown in Figure 1, indicates that one type of information conveyed via urine to the male gerbil in a laboratory setting might be sexual identification. Additionally, the finding that the strongest reaction was toward urine from female gerbils in estrus suggests that urine may be used by the male gerbil in the identification of the female's sexual state.

Female gerbils did not respond differentially to any particular urine sample, possibly because females do not utilize the information contained in urine to the extent male gerbils do. Female subjects have not specifically been tested for reactions to urine in either rats or guinea pigs, while, in mice, Doty (1973) found that one species tended to prefer male mouse urine, while a second did not.

Many of the times spent near a stimulus for males, and especially for females, were close to or slightly less than $30 \mathrm{sec}$ (the amount of time expected with random movement about the cage by the subject). Therefore it might be argued that the subjects were actually avoiding the stimulus. The Friedman test showed, however, that male subjects definitely preferred the stimulus end of the cage whenever female gerbil urine was present, and under no condition was either sex found to spend significantly more time in the opposite end of the cage.

Each comparison was analyzed separately. However, the time spent with any particular urine stimulus was fairly consistent among comparisons for each sex group. For example, male subjects spent about the same amount of time in the section containing female gerbil urine, irrespective of the second stimulus condition. Thus, the subjects might have been responding to each stimulus independently rather than as pairs.

The findings of the current study indicate that, at least in the laboratory, female gerbil urine conveys sexual information, including sexual identity and especially sexual readiness, to the male gerbil. Adding to the findings of Dagg and Windsor (1971) and Halpin (1974) that gerbils are able to discriminate between the urine from two male gerbils (individual identification), this study contributed to a better understanding of the olfactory social communication system in the gerbil.

\section{REFERENCES}

BARAN, D. Responses of male Mongolian gerbils to male gerbil odors. Journal of Comparative and Physiological Psychology, 1973, 84, 63-72

Barfield, M. A..\& BeEman. E. A. Oestrous cycle in Mongolian gerbil Meriones unguiculatus. Journal of Reproduction and Fentility. 1968, 17, 247-250.

Beauchamp, G. K. Attraction of male guinea pigs to conspecific urine. Physiology and Behavior. 1973, 10, 589-594.

Calhoun. J. B. The ecology and sociology of the Norway rat Washington, D.C: Government Printing Oftice, 1962.

DAgG, A. I., \& WINDsor, D. E. Olfactory discrimination limits in gerbils. Canadian Joumal of Zoology, 1971, 49, 283-285.

Doty, R. L. Reactions of deer mice (Peromyscus maniculatus) and white-footed mice (Peromyscus leucopus) to homospecitic and heterospecitic urine odors. Journal of Comparative and Physiological Psychology. 1973, 84. 296-303.

HaLPIN, Z. T. Individual differences in the biological odors of the Mongolian gerbil (Meriones unguiculatus). Behavioral Biology, 1974, 11. 253-259.

LydelL, K., \& Doty, R. L. Male rat odor preferences for female urine as a function of sexual experience, urine age, and urine source. Hormones and Behavior, 1972, 3, 205-212.

Nyby, J., Thiessen, D. D., \& Wallace, P. Social inhibition of territorial marking in the Mongolian gerbil (Meriones unguiculutus). Psychonomic Science, 1970, 21. 310-312.

Thiessen, D. D., Friend, H. C., \& Lindzey, G. Androgen control of territorial marking in the Mongolian gerbil. Science, 1968. 160. $432-434$.

(Received for publication October 28, 1976; revision accepted June 8,1977 .) 